\title{
Ternary Ladder Operators
}

Benedict Irwin ${ }^{1}$

${ }^{1}$ Affiliation not available

November 2, 2020

\section{Abstract}

We develop a triplet operator system which encompasses the structure of quark combinations. Ladder operators are created. The constants $\beta$ are currently being found.

\section{Ternary Ladders}

Based on quarks

Define:

$\mathrm{a}-\alpha>=\beta_{a}^{\alpha} \mid \alpha-\frac{1}{3}>$

$a^{+}\left|\alpha>=\beta_{a^{+}}^{\alpha}\right| \alpha+\frac{2}{3}>$

$-\alpha>=\beta_{\hat{a}}^{\alpha} \mid \alpha+\frac{1}{3}>$

$\hat{a}^{+}\left|\alpha>=\beta_{\hat{a}^{+}}^{\alpha}\right| \alpha-\frac{2}{3}>$ (1)

So in Triplet operations exists operations which have an eigenstate of $\mid \alpha>$ and counting triplets and also binary operations in the same way quarks combine.

$\mathrm{aaa}^{+}\left|\alpha>=\beta_{a^{+}}^{\alpha} \beta_{a}^{\alpha+\frac{2}{3}} \beta_{a}^{\alpha+\frac{1}{3}}\right| \alpha>$

$a^{+} a^{+} a\left|\alpha>=\beta_{a}^{\alpha} \beta_{a^{+}}^{\alpha-\frac{1}{3}} \beta_{a^{+}}^{\alpha+\frac{1}{3}}\right| \alpha+1>$

aaa $\left|\alpha>=\beta_{a}^{\alpha} \beta_{a}^{\alpha-\frac{1}{3}} \beta_{a}^{\alpha-\frac{2}{3}}\right| \alpha-1>$

$a^{+} a^{+} a^{+}\left|\alpha>=\beta_{a^{+}}^{\alpha} \beta_{a^{+}}^{\alpha+\frac{2}{3}} \beta_{a^{+}}^{\alpha+\frac{4}{3}}\right| \alpha+2>$

So considering the $\beta$ terms. The only time $\beta_{a}^{\alpha-\frac{2}{3}}$ might arise is if an a has operated on a state that is already filled by $\alpha-\frac{2}{3}$. So, it should seem appropriate that if $\alpha=0$ this coeficcient drops to zero to prevent negative integer fillings. This proposes a trial function of 
$\beta_{a}^{\alpha}=\sqrt[3]{\frac{2}{3}+\alpha}(3)$

Then the operation

aaa $-\alpha>=\sqrt[3]{\frac{2}{3}+\alpha} \sqrt[3]{\frac{2}{3}+\alpha-\frac{1}{3}} \sqrt[3]{\frac{2}{3}+\alpha-\frac{2}{3}} \mid \alpha-1>(4)$

and for $\alpha=0$ the coefficient drops to zero.

One could forsee that if the is a base number operator $N=a a a^{+}$then combinations of these transitions could be such that

(aaa) $\left(\mathrm{a}^{+} a^{+} a\right)\left|\alpha>=a\left(a a a^{+}\right) a^{+} a\right| \alpha>(5)$

For doublet (meson) operations take an operator and it's anti-operator

$+a^{+}\left|\alpha>=\beta_{a^{+}}^{\alpha} \beta_{\hat{a}^{+}}^{\alpha+\frac{2}{3}}\right| \alpha>$

$a^{+} \hat{a}^{+}\left|\alpha>=\beta_{\hat{a}^{+}}^{\alpha} \beta_{a^{+}}^{\alpha-\frac{2}{3}}\right| \alpha>$

(6)

Question? Does

$$
\beta_{a^{+}}^{\alpha} \beta_{\hat{a}^{+}}^{\alpha+\frac{2}{3}}=\beta_{\hat{a}^{+}}^{\alpha} \beta_{a^{+}}^{\alpha-\frac{2}{3}} ?
$$

\section{Another Method}

Another method to try is 3 (or 6 really) operators, for example

$\mathrm{a}_{+}^{r}|\alpha>=\sqrt[3]{\alpha}| \alpha+\frac{2}{3}>$

$a_{-}^{r}|\alpha>=\sqrt[3]{\alpha}| \alpha-\frac{1}{3}>$

$a_{+}^{g}\left|\alpha>=\sqrt[3]{\alpha-\frac{1}{3}}\right| \alpha+\frac{2}{3}>$

$a_{-}^{g}\left|\alpha>=\sqrt[3]{\alpha-\frac{1}{3}}\right| \alpha-\frac{1}{3}>$

$a_{+}^{b}\left|\alpha>=\sqrt[3]{\alpha+\frac{1}{3}}\right| \alpha+\frac{2}{3}>$

$a_{-}^{b}\left|\alpha>=\sqrt[3]{\alpha+\frac{1}{3}}\right| \alpha-\frac{1}{3}>$

Then is was found that the combination

$\mathrm{a}_{+}^{r} a_{-}^{g} a_{+}^{b}\left|\alpha>=\left(\alpha+\frac{1}{3}\right)\right| \alpha+1>(9)$ 


\section{Stable Number Operator}

An eigenstate for a ternay triplet was found. Define:

$\mathrm{r}^{+}|\alpha>=\sqrt[3]{\alpha}| \alpha+\frac{2}{3}>$

$r|\alpha>=\sqrt[3]{\alpha}| \alpha-\frac{1}{3}>$

$g^{+}\left|n>=\sqrt[3]{n+\frac{2}{3}}\right| n+\frac{2}{3}>$

$g\left|\alpha>=\sqrt[3]{\alpha-\frac{2}{3}}\right| \alpha-\frac{1}{3}>$

$b^{+}\left|n>=\sqrt[3]{n+\frac{1}{3}}\right| n+\frac{2}{3}>$

$b\left|\alpha>=\sqrt[3]{\alpha-\frac{1}{3}}\right| \alpha-\frac{1}{3}>(10)$

Then the operation

$a_{-}^{b} a_{-}^{g} a_{+}^{r}\left|\alpha>=a_{-}^{b} a_{-}^{g} \sqrt[3]{\alpha}\right| \alpha+\frac{2}{3}>$

$=a_{-}^{b} \sqrt[3]{\alpha} \sqrt[3]{\alpha} \mid \alpha+\frac{1}{3}>$

$a_{-}^{b} a_{-}^{g} a_{+}^{r}|\alpha>=\alpha| \alpha>(11)$

and then acts like a number operator

Then there are operations:

$$
a^{r} a^{b} a^{g}\left|\alpha>=\left(\alpha-\frac{2}{3}\right)\right| \alpha-1>\ldots b g r \mid \alpha>=
$$

Hamiltonian basic version:

$\mathrm{H}=\mathrm{Wbgr}^{+}$

(13)

TERNARY COMMUTATOR? $[\mathrm{a}, \mathrm{b}, \mathrm{c}]=\mathrm{abc}-\mathrm{acb}-\mathrm{bac}+\mathrm{bca}+\mathrm{cab}-\mathrm{cba}$

\begin{tabular}{|c|c|}
\hline combin. & coeff \\
\hline$b g r^{+}$ & $\alpha$ \\
$b r^{+} g$ & $\sqrt[3]{\alpha} \sqrt[3]{\alpha-\frac{1}{3}} \sqrt[3]{\alpha-\frac{2}{3}}$ \\
$g b r^{+}$ & $\sqrt[3]{\alpha} \sqrt[3]{\alpha+\frac{1}{3}} \sqrt[3]{\alpha-\frac{1}{3}}$ \\
$g r^{+} b$ & $\alpha-\frac{1}{3}$ \\
$r^{+} b g$ & $\alpha-\frac{2}{3}$ \\
$r^{+} g b$ & $\sqrt[3]{\alpha-1} \sqrt[3]{\alpha-\frac{1}{3}} \sqrt[3]{\alpha-\frac{2}{3}}$ \\
\hline
\end{tabular}

Thus

$$
\left[b, g, r^{+}\right]=\alpha-\sqrt[3]{\alpha} \sqrt[3]{\alpha-\frac{1}{3}} \sqrt[3]{\alpha-\frac{2}{3}}-\sqrt[3]{\alpha} \sqrt[3]{\alpha+\frac{1}{3}} \sqrt[3]{\alpha-\frac{1}{3}}+\left(\alpha-\frac{1}{3}\right)+\left(\alpha-\frac{2}{3}\right)-\sqrt[3]{\alpha-1} \sqrt[3]{\alpha-\frac{1}{3}} \sqrt[3]{\alpha-\frac{2}{3}}
$$


Out of all possible operators involving 3 of the primitive operators above there are 10 solutions with "nice" coefficients. 1 behaves as an annihilation operator, 3 behave as creation operators and the others are like number operators and thus eigen operators of the states.

\begin{tabular}{|c|c|}
\hline$r^{+} b g \mid n>$ & $\left(n-\frac{2}{3}\right) \mid n>$ \\
$r^{+} b b^{+} \mid n>$ & $\left(n+\frac{1}{3}\right) \mid n+1>$ \\
$r b g \mid n>$ & $\left(n-\frac{2}{3}\right) \mid n-1>$ \\
$r b b^{+} \mid n>$ & $\left(n+\frac{1}{3}\right) \mid n>$ \\
$g r^{+} g^{+} \mid n>$ & $\left(n+\frac{2}{3}\right) \mid n+1>$ \\
$g r^{+} b \mid n>$ & $\left(n-\frac{1}{3}\right) \mid n>$ \\
$b^{+} r g^{+} \mid n>$ & $\left(n+\frac{2}{3}\right) \mid n+1>$ \\
$b^{+} r b \mid n>$ & $\left(n-\frac{1}{3}\right) \mid n>$ \\
$b g r^{+} \mid n>$ & $n \mid n>$ \\
$b b^{+} r \mid n>$ & $n \mid n>$ \\
\hline
\end{tabular}

\section{Table of 3 operations}

This is an exhaustive table of operations invlolving three of the above defined operators 


\begin{tabular}{|c|c|}
\hline$r^{+} r^{+} r^{+}$ & $\sqrt[3]{n+\frac{0}{3}} \sqrt[3]{n+\frac{2}{3}} \sqrt[3]{n+\frac{4}{3}}$ \\
\hline$r^{+} r^{+} r$ & $\sqrt[3]{n+\frac{0}{3}} \sqrt[3]{n-\frac{1}{3}} \sqrt[3]{n+\frac{1}{3}}$ \\
\hline$r^{+} r^{+} g^{+}$ & $\sqrt[3]{n+\frac{2}{3}} \sqrt[3]{n+\frac{2}{3}} \sqrt[3]{n+\frac{4}{3}}$ \\
\hline$r^{+} r^{+} g$ & $\sqrt[3]{n-\frac{2}{3}} \sqrt[3]{n-\frac{1}{3}} \sqrt[3]{n+\frac{1}{3}}$ \\
\hline$r^{+} r^{+} b^{+}$ & $\sqrt[3]{n+\frac{1}{3}} \sqrt[3]{n+\frac{2}{3}} \sqrt[3]{n+\frac{4}{3}}$ \\
\hline$r^{+} r^{+} b$ & $\sqrt[3]{n-\frac{1}{3}} \sqrt[3]{n-\frac{1}{3}} \sqrt[3]{n+\frac{1}{3}}$ \\
\hline$r^{+} r r^{+}$ & $\sqrt[3]{n+\frac{0}{3}} \sqrt[3]{n+\frac{2}{3}} \sqrt[3]{n+\frac{1}{3}}$ \\
\hline$r^{+} r r$ & $\sqrt{n-\frac{1}{3}} \sqrt[3]{n-\frac{2}{3}}$ \\
\hline$r^{+} \mathrm{rg}^{+}$ & $n+\frac{2}{3} \sqrt[3]{n+\frac{1}{3}}$ \\
\hline$r^{+} r g$ & $n-\frac{1}{3} \sqrt[3]{n-\frac{2}{3}}$ \\
\hline$r^{+} r b^{+}$ & $\sqrt{n+\frac{2}{3}} \sqrt[3]{n+\frac{1}{3}}$ \\
\hline$r^{+} r b$ & $n-\frac{1}{3} \sqrt[3]{n-\frac{2}{3}}$ \\
\hline$r^{+} g^{+} r^{+}$ & $n+\frac{4}{3} \sqrt[3]{n+\frac{4}{3}}$ \\
\hline$r^{+} g^{+} r$ & $n+\frac{1}{3} \sqrt[3]{n+\frac{1}{3}}$ \\
\hline$r^{+} g^{+} g^{+}$ & $\sqrt[3]{n+\frac{4}{3}} \sqrt[3]{n+\frac{4}{3}}$ \\
\hline$r^{+} g^{+} g$ & $n+\frac{1}{3} \sqrt[3]{n+\frac{1}{3}}$ \\
\hline$r^{+} g^{+} b^{+}$ & $\sqrt{n+\frac{4}{3}} \sqrt[3]{n+\frac{4}{3}}$ \\
\hline$r^{+} g^{+} b$ & $\sqrt[3]{n-\frac{1}{3}} \sqrt[3]{n+\frac{1}{3}} \sqrt[3]{n+\frac{1}{3}}$ \\
\hline$r^{+} g r^{+}$ & $n+\frac{0}{3} \sqrt[3]{n+\frac{1}{3}}$ \\
\hline$r^{+} g r$ & $\sqrt[3]{n+\frac{0}{3}} \sqrt[3]{n-\frac{3}{3}} \sqrt[3]{n-\frac{2}{3}}$ \\
\hline$r^{+} g g^{+}$ & $\sqrt[3]{n+\frac{2}{3}} \sqrt[3]{n+\frac{0}{3}} \sqrt[3]{n+\frac{1}{3}}$ \\
\hline$r^{+} g g$ & $\sqrt[3]{n-\frac{2}{3}} \sqrt[3]{n-\frac{3}{3}} \sqrt[3]{n-\frac{2}{3}}$ \\
\hline$r^{+} g b^{+}$ & $\sqrt[3]{n+\frac{1}{3}} \sqrt[3]{n+\frac{0}{3}} \sqrt[3]{n+\frac{1}{3}}$ \\
\hline$r^{+} g b$ & $\sqrt[3]{n-\frac{3}{3}} \sqrt[3]{n-\frac{2}{3}}$ \\
\hline$r^{+} b^{+} r^{+}$ & $\sqrt[3]{n+\frac{0}{3}} \sqrt[3]{n+\frac{3}{3}} \sqrt[3]{n+\frac{4}{3}}$ \\
\hline$r^{+} b^{+} r$ & $\sqrt[3]{n+\frac{0}{3}} \sqrt[3]{n+\frac{0}{3}} \sqrt[3]{n+\frac{1}{3}}$ \\
\hline$r^{+} b^{+} g^{+}$ & $\sqrt[3]{n+\frac{2}{3}} \sqrt[3]{n+\frac{3}{3}} \sqrt[3]{n+\frac{4}{3}}$ \\
\hline$r^{+} b^{+} g$ & $\sqrt[3]{n-\frac{2}{3}} \sqrt[3]{n+\frac{0}{3}} \sqrt[3]{n+\frac{1}{3}}$ \\
\hline$r^{+} b^{+} b^{+}$ & $\sqrt[3]{n+\frac{1}{3}} \sqrt[3]{n+\frac{3}{3}} \sqrt[3]{n+\frac{4}{3}}$ \\
\hline$r^{+} b^{+} b$ & $\sqrt[3]{n-\frac{1}{3}} \sqrt[3]{n+\frac{0}{3}} \sqrt[3]{n+\frac{1}{3}}$ \\
\hline$r^{+} b r^{+}$ & $\sqrt[3]{n+\frac{0}{3}} \sqrt[3]{n+\frac{1}{3}} \sqrt[3]{n+\frac{1}{3}}$ \\
\hline$r^{+} b r$ & $\sqrt[3]{n+\frac{0}{3}} \sqrt[3]{n-\frac{2}{3}} \sqrt[3]{n-\frac{2}{3}}$ \\
\hline$r^{+} b g^{+}$ & $\sqrt[3]{n+\frac{2}{3}} \sqrt[3]{n+\frac{1}{3}} \sqrt[3]{n+\frac{1}{3}}$ \\
\hline$r^{+} b g$ & $\sqrt[3]{n-\frac{2}{3}} \sqrt[3]{n-\frac{2}{3}} \sqrt[3]{n-\frac{2}{3}}$ \\
\hline$r^{+} b b^{+}$ & $\sqrt[3]{n+\frac{1}{3}} \sqrt[3]{n+\frac{1}{3}} \sqrt[3]{n+\frac{1}{3}}$ \\
\hline$r^{+} b b$ & $\sqrt[3]{n-\frac{1}{3}} \sqrt[3]{n-\frac{2}{3}} \sqrt[3]{n-\frac{2}{3}}$ \\
\hline$r r^{+} r^{+}$ & $\sqrt[3]{n+\frac{0}{3}} \sqrt[3]{n+\frac{2}{3}} \sqrt[3]{n+\frac{4}{3}}$ \\
\hline$r r^{+} r$ & $\sqrt[3]{n+\frac{0}{3}} \sqrt[3]{n-\frac{1}{3}} \sqrt[3]{n+\frac{1}{3}}$ \\
\hline$r r^{+} g^{+}$ & $\sqrt[3]{n+\frac{2}{3}} \sqrt[3]{n+\frac{2}{3}} \sqrt[3]{n+\frac{4}{3}}$ \\
\hline
\end{tabular}




\section{$6 \quad$ Using Permutations}

So a nice trick with the values from the table would be to construct an operator

$\mathrm{O}=2 \mathrm{bgr}^{+}-g r^{+} b-r^{+} b g(18)$ such that

$$
O\left|\alpha>=\left[2 \alpha-\alpha+\frac{1}{3}-\alpha+\frac{2}{3}\right]\right| \alpha>=\mid \alpha>
$$

Then we have our TERNARY commutator equivalent

$\mathrm{O}=2 \mathrm{bgr}^{+}-g r^{+} b-r^{+} b g=\left[b, g, r^{+}\right]=1(20)$

It appears to be permutation based. So we require the properties of such a mathematical device.

$$
[a, b, c]=2 a b c-c a b-b c a
$$

Using the rules above, a change in permutation would go like

$$
\left[b, g, r^{+}\right]=1
$$

g, $\mathrm{r}^{+}, b$

$=0$

$\mathrm{r}^{+}, b, g$

$=-1$

$(22)$

So there are three permutations/rotations of these variables. And the three ternary as opposed to binary outputs $-1,0,1$. Very exciting. Therefore we have

$$
\left[b, g, r^{+}\right]+\left[r^{+}, b, g\right]=\left[g, r^{+}, b\right]=0
$$

If one switches two of the values side by side then it will lead to the complicated cube root coeficcients, it is not obvious if they cancel out under the same rules. We seek some mathematical differences and similarities between this contstruction and the regular binary commutator, for example, for some constant $g$

$[\mathrm{ga}, \mathrm{b}, \mathrm{c}]=2 \mathrm{gabc}-\mathrm{cgab}-\mathrm{bcga}=\mathrm{g}(2 \mathrm{abc}-\mathrm{cab}-\mathrm{bca})=\mathrm{g}[\mathrm{a}, \mathrm{b}, \mathrm{c}](24)$ 
As an algebraic property the summation results of the commutator were partially responsible

$$
[a, b, c]=2 a b c-c a b-b c a=2 b c a-a b c-c a b=2 c a b-b c a-a b c
$$

$\mathrm{b}, \mathrm{c}, \mathrm{a}$

$c, a, b$

Thus

$$
[a, b, c]+[b, c, a]+[c, a, b]=0
$$

Another fantastic property is that

$[\mathrm{a}, \mathrm{b}, 1]=2 \mathrm{ab}-\mathrm{ab}-\mathrm{ba}=\mathrm{ab}-\mathrm{ba}=[\mathrm{a}, \mathrm{b}](27)$

amazing!, it restores the binary commutator if only two non commuting variables enter. another key result is

$[\mathrm{a}, \mathrm{b}+\mathrm{x}, \mathrm{c}]=[\mathrm{a}, \mathrm{b}, \mathrm{c}]+[\mathrm{a}, \mathrm{x}, \mathrm{c}]$

For expansions such as

$[\mathrm{a}, \mathrm{bx}, \mathrm{c}]=\mathrm{b}[\mathrm{a}, \mathrm{x}, \mathrm{c}]+[\mathrm{a}, \mathrm{b}, \mathrm{c}] \mathrm{x}$

$(29)$

to be true, then there must exist the identity

$a b c x+b a x c-b c a x-a b x c=0(30)$

For

$[\mathrm{a}, \mathrm{b}, \mathrm{cx}]=\mathrm{c}[\mathrm{a}, \mathrm{b}, \mathrm{x}]+[\mathrm{a}, \mathrm{b}, \mathrm{c}] \mathrm{x}(31)$

It must be set that

cabx + bcxa-bcax-cbxa $=0(32)$ 


\section{Runthrough of normal QFT}

So this commutator should work with the old algebras, for example bosonic

$\mathrm{a}-\mathrm{n} \dot{i}=\sqrt{n} \mid n-1>$

$a^{+}|n>=\sqrt{n+1}| n+1>(33)$

we know that

$\left[\mathrm{a}, \mathrm{a}^{+}\right]=1=\left[a, a^{+}, 1\right](34)$

So trying

$$
\left[a, a^{+}, a\right]|n>=2 n \sqrt{n}| n-1>-(n+1) \sqrt{n}|n-1>-(n-1) \sqrt{n}| n-1>=0
$$

Meaning

$2 \mathrm{aa}^{+} a=a a a^{+}+a^{+} a a(36)$

Deep representation concept, if $a a^{+} a$ were equivalent to cos then $a a a^{+}$is equivalent to $\exp (i \ldots)$ and $a^{+} a a$ is equivalent to $\exp (-i \ldots)$ then $i \sin$ is equivalent to $a$ as is cos as operators. Then this is a kind of rotation.

Some relationships

$\left[\mathrm{a}, \mathrm{a}^{+}, 1\right]=2 a a^{+}-a a^{+}-a^{+} a=\left[a, a^{+}\right]$

$\mathrm{a}^{+}, a, 1$

$=2 a^{+} a-a^{+} a-a a^{+}=\left[a^{+}, a\right]$

$(37)$

\section{Fermion Combinations}

Try combination

$\mathrm{r}^{+}\left|n>=\sqrt[3]{1-\left(n-\frac{3}{3}\right)}\right| n+\frac{2}{3}>$

$r|n>=\sqrt[3]{n}| n-\frac{1}{3}>$

$b^{+}\left|n>=\sqrt[3]{1-\left(n-\frac{1}{3}\right)}\right| n+\frac{2}{3}>$

$b\left|n>=\sqrt[3]{n-\frac{1}{3}}\right| n-\frac{1}{3}>$

$g^{+}\left|n>=\sqrt[3]{1-\left(n-\frac{2}{3}\right)}\right| n+\frac{2}{3}>$

$g\left|n>=\sqrt[3]{n-\frac{2}{3}}\right| n-\frac{1}{3}>$

(38)

The number operator is then

$\operatorname{bgr}^{+}|n>=\sqrt[3]{n} \sqrt[3]{n} \sqrt[3]{1-(n-1)}| n>(39)$ 
which conforms to fermionic states filled with 1 or 0.

Then upon the addition of one quantum

$$
b g^{+} r^{+}=\sqrt[3]{n+1} \sqrt[3]{1-n} \sqrt[3]{1-(n-1)} \mid n+1>
$$

allowing filling into the ground state with coefficient $\sqrt[3]{2}$ but not an already filled state.

The removal of one quantum may correspond to the operator

$\mathrm{rbg}-\mathrm{n} \dot{i}=\left(\mathrm{n}-2 \frac{}{3) \mid n-1>(41)}\right.$ Which has a positive probability for a filled state and surprisingly a negative (probabilty? coeff, rate?) for an unfilled state... Interpretation of this... possibly

There would also exist a double jump operation as

$\mathrm{b}^{+} g^{+} r^{+}|n>=\sqrt[3]{1-(n+1)} \sqrt[3]{1-n} \sqrt[3]{1-(n-1)}| n+2>(42)$

But this has a zero coeficcient for either $n=1$ or $n=0$.

There are also single "colour" operations such as

$\operatorname{rrr}-\mathrm{n}_{i}=\sqrt[3]{n} \sqrt[3]{n-\frac{1}{3}} \sqrt[3]{n-\frac{2}{3}} \mid n-1>$

which can only happen in a filled state. Try ternary anti-commutator $\{a, b, c\}=a b c+a c b+b a c+b c a+c a b+c b a$ seek it equals 1.

\section{Further n-utator Work}

So there is a general trend behing the commutators, such that an $\mathrm{n}$-utator, that is a $[\mathrm{x}, \mathrm{y}, \ldots, \mathrm{z}]$ construction with $n$ elements is equal to (n-1) lots of the principle permutation, minus 1 of each other permutation. that is

$\left[\mathrm{x}_{1}, x_{2}, \ldots, x_{N}\right]=(N-1) x_{1} x_{2} \ldots x_{N-1} x_{N}-x_{N} x_{1} x_{2} \ldots x_{N-1}-x_{N-1} x_{N} \ldots x_{1} x_{2}-\ldots-x_{2} \ldots x_{N-1} x_{N} x_{1}$

Which results in any element being a constant, in a reduction to a lower N-utator. Some examples:

$[\mathrm{a}]=0 \mathrm{a}$ 
$\mathrm{a}, \mathrm{b}$

$=1 \mathrm{ab}-\mathrm{ba}$

a,b,c

$=2 \mathrm{abc}-\mathrm{bca}-\mathrm{cab}$

a,b,c,d

$=3 a b c d-$ dabc - cdab - bcda

a,b,c,d,e

$=4 a b c d e-$ eabcd - deabc - cdeab - bcdea

(45) 